\title{
Ideal boundary of 7-systolic complexes and groups
}

\author{
DAMIAN OSAJDA
}

\begin{abstract}
We prove that ideal boundary of a 7-systolic group is strongly hereditarily aspherical. For some class of 7-systolic groups we show their boundaries are connected and without local cut points, thus getting some results concerning splittings of those groups.
\end{abstract}

20F65; 20F69

\section{Introduction}

The notion of a $k$-systolic complex ( $k$ a natural number at least 6 ) was introduced by T Januszkiewicz and J Świątkowski [13] and, independently, by F Haglund [10] as a combinatorial analogue of a nonpositively curved space. A $k$-systolic complex is a simply connected simplicial complex satisfying some local combinatorial conditions. Roughly speaking, there is a lower bound for the length of "essential" closed paths in a one-skeleton of every link.

A group acting geometrically by automorphisms on a $k$-systolic complex is called a $k$-systolic group. Januszkiewicz and Świastkowski constructed examples of such torsion free groups of arbitrary large cohomological dimension, for every $k \geq 6$ [13]. The examples are fundamental groups of some simplices of groups. In the same paper they prove that 7-systolic groups are Gromov-hyperbolic.

In this paper we study 7 -systolic complexes and groups and in particular their ideal boundaries. Our main result is the following.

Main Theorem (Theorem 4.2) The ideal boundary of a 7-systolic group is a strongly hereditarily aspherical compactum.

The notion of strong hereditary asphericity (see Section 2.3 for the precise definition) was introduced by R J Daverman [5]. Roughly speaking, a space is hereditarily aspherical if each of its closed subsets is aspherical. The significance of this notion follows from the fact that a cell-like map defined on a strongly hereditarily aspherical compactum does not raise dimension. 
The Main Theorem shows that 7-systolic groups are quite different from many classical hyperbolic groups. It gives also new examples of topological spaces that can occur as boundaries of hyperbolic groups. The question about the spaces being such boundaries is well understood only in dimensions 0 and 1 - compare Kapovich and Kleiner [16]. It is still not known which topological spaces can be higher dimensional boundaries of hyperbolic groups and only a few homeomorphism types of such spaces are known-see Benakli and Kapovich [15] and Remarks 4.3 (1). Moreover, we show in Corollary 5.7 that for certain classes of complexes (and groups) their ideal boundaries are "simple" in a sense-they are connected and have no local cut points.

In order to prove the main theorem we define (in Section 3) an inverse system of combinatorial spheres in a 7-systolic complex and projections onto them, whose inverse limit is the ideal boundary of the complex. It should be noticed that even in the more general systolic (which means 6-systolic) case some such inverse system can be defined (cf Januszkiewicz and Świątkowski [13, Section 8]). However its properties prevent it from being the right tool to define a reasonable boundary of a systolic complex or group. In particular the inverse limit of this standard systolic inverse system in a 7 -systolic case is not the Gromov boundary. On the other hand, our construction is not valid in general systolic case, although some results are probably true for hyperbolic systolic (not necessarily 7-systolic) groups.

In Section 5 we study further properties of boundaries of some 7-systolic complexes. In particular we prove the following theorem, which is a special case of Theorem 5.6 proved in that section.

Theorem (Corollary 5.7) Let $X$ be a locally finite 7-systolic normal pseudomanifold of dimension at least 3 . Then its ideal boundary $\partial X$ is connected and has no local cut points.

Via the results of Stallings [19] and the ones of Bowditch [2] the latter theorem implies the following.

Theorem (Corollary 5.9) A group acting geometrically by automorphisms on a locally finite 7-systolic normal pseudomanifold of dimension at least 3 does not split essentially over a finite or two-ended group as an amalgamated product or an $H N N$-extension.

Groups acting geometrically on such pseudomanifolds of arbitrary large dimension were constructed in Januszkiewicz and Świątkowski [13] and are the only 7-systolic groups of cohomological dimension greater than 2 known to us at the moment.

Algebraic 83 Geometric Topology, Volume 8 (2008) 
Acknowledgements I would like to thank Tadeusz Januszkiewicz and Jacek Świątkowski for introducing me to the subject and suggesting the problem. I am also grateful to them and to Craig Guilbault, Krzysztof Omiljanowski and Carrie Schermetzler for helpful conversations.

The author was a Marie Curie Intra-European fellow, contract MEIF CT 05011050 and was partially supported by the Polish Scientific Research Committee (KBN) grant 2 P03A 01725.

\section{Preliminaries}

\subsection{Simplicial complexes}

In this section we recall some definitions and fix the notation.

Let $X$ be a simplicial complex. We denote by $X^{\prime}$ the first barycentric subdivision of $X$. For a natural number $k$, we denote by $X^{(k)}$ the $k$-skeleton of $X$, ie the union of all simplices of $X$, of dimension at most $k$. For a given subset $C=\left\{v_{1}, v_{2}, \ldots, v_{l}\right\}$ of $X^{(0)}$ we denote by $\left\langle v_{1}, v_{2}, \ldots, v_{l}\right\rangle$ the minimal simplex in $X$ containing $C$-the simplex spanned by $C$. We denote by $X_{\sigma}$ the link of a given simplex $\sigma$ in $X$. A simplicial complex $X$ is flag if every set $B$ of pairwise connected (by edges) vertices of $X$ spans a simplex in $X$.

Recall that a subcomplex $Y$ of $X$ is full if every set $B$ of vertices of $Y$ spanning a simplex of $X$ spans a simplex in $Y$. We denote by $\sigma * \rho$ the join of simplices $\sigma$ and $\rho$.

If it is not stated otherwise a simplicial complex $X$ is equipped with a path metric $d_{X}$ for which every $k$-simplex of $X$ is isometric to the regular Euclidean $k$-simplex.

A simplicial complex $X$ is called a chamber complex of dimension $n$ if it is the union of $n$-simplices (which are called chambers of $X$ ) and for every $(n-1)$-dimensional face of $X$ there exist at least two chambers containing that face. It is easy to see that links in a chamber complex are themselves chamber complexes. A gallery in a chamber complex is a finite sequence of maximal simplices such that two consecutive simplices share a common face of codimension 1 . A chamber complex is said to be gallery connected if any two chambers can be connected by a gallery. Chamber complex is normal if it is gallery connected and all its links of dimension above 0 are gallery connected. A chamber complex is a pseudomanifold if every codimension one face belongs to exactly two chambers.

Algebraic $8 \mathcal{G}$ Geometric Topology, Volume 8 (2008) 


\subsection{Systolic complexes and groups}

We follow here Januszkiewicz and Świątkowski [13; 14]. For a given natural number $k \geq 4$, a simplicial complex $X$ is $k$-large if it is flag and every cycle $\gamma$ in $X$ (ie a subcomplex homeomorphic to the circle) of length $4 \leq|\gamma|<k$ has a diagonal (ie an edge connecting two nonconsecutive vertices in $\gamma$ ). Here $|\gamma|$ denotes the number of edges of $\gamma$.

A simplicial complex $X$ is locally $k$-large if for every simplex $\sigma \neq \varnothing$ of $X$ its link $X_{\sigma}$ in $X$ is $k$-large.

$X$ is $k$-systolic if it is locally $k$-large, connected and simply connected.

Because $k=6$ is of special importance in that theory, 6-systolic complexes are called systolic.

A group acting geometrically (ie properly discontinuously and cocompactly) by simplicial automorphisms on a $k$-systolic (resp. systolic) complex is called $k$-systolic (resp. systolic). Free groups and fundamental groups of surfaces are systolic groups. In Januszkiewicz and Świątkowski [13], for arbitrary $k$ and $n$, a torsion free $k$-systolic group of cohomological dimension $n$ is constructed. Those groups are the fundamental groups of some simplices of groups.

In the rest of this subsection we list some results concerning systolic complexes and groups. We begin with the easy facts whose proofs can be found in [13].

Proposition 2.1 (1) If $k>m$ and $X$ is $k$-large then $X$ is also $m$-large.

(2) A full subcomplex in a (locally) $k$-large complex is (locally) $k$-large.

(3) Links of a $k$-large complex are $k$-large.

(4) There is no $k$-large triangulation of the $2-$ sphere for $k \geq 6$. Hence no triangulation of a manifold of dimension above 2 is 6 -large since 2 -spheres occur as links of some simplices in that case.

The following property of 7 -systolic complexes is crucial for this paper.

Theorem 2.2 [13, Theorem 2.1] Let $X$ be a 7-systolic complex. Then the 1skeleton of $X$ with its standard geodesic metric is $\delta$-hyperbolic with $\delta=2 \frac{1}{2}$.

Thus 7-systolic groups are word-hyperbolic.

In [13, Sections 3 and 7] the notion of a convex subcomplex of a systolic complex is introduced. A simplex is a convex subcomplex.

Algebraic 83 Geometric Topology, Volume 8 (2008) 
For a simplicial complex $X$ and its subcomplex $Q$ we can define a closed combinatorial ball of radius $i$ around $Q$ in $X, B_{i}(Q, X)$, inductively: $B_{0}(Q, X)=Q$ and $B_{i}(Q, X)=\bigcup\left\{\tau: \tau \cap B_{i-1}(Q, X) \neq \varnothing\right\}$, for every positive natural number $i$.

By $S_{i}(Q, X)$ we denote the subcomplex of $B_{i}(Q, X)$ spanned by the vertices at combinatorial distance $i$ from $Q$, ie not belonging to $B_{i-1}(Q, X)$ (for $i>0$ ). We denote by $\stackrel{\circ}{B}_{i}(Q, X)$ the interior of the closed combinatorial $i$-ball around $\sigma$ in $X$, ie $\stackrel{\circ}{B}_{i}(Q, X)=B_{i}(Q, X) \backslash S_{i}(Q, X)$.

For the rest of this section let $X$ denote a systolic complex and $Q$ its convex subcomplex.

Lemma 2.3 [13, Lemmas 7.5 and 7.6] The sphere $S_{i}(Q, X)$ and the ball $B_{i}(Q, X)$ are full subcomplexes of $X$ and they are 6-large.

Lemma 2.4 [13, Section 7] If $i>0$, then for every simplex $\tau \in S_{i}(Q, X), \rho=$ $\partial B_{i-1}(Q, X) \cap X_{\tau}$ is a single simplex, $X_{\tau} \cap B_{i}(Q, X)=B_{1}\left(\rho, X_{\tau}\right)$ and $X_{\tau} \cap$ $S_{i}(Q, X)=S_{1}\left(\rho, X_{\tau}\right)$.

In the rest of the paper we call the simplex $\rho$, as in the above lemma the projection of $\tau$ on $S_{i-1}(Q, X)$.

The universal cover of a connected locally 6-large simplicial complex is systolic and the following fact holds.

Theorem 2.5 [13, Theorem 4.1] The universal cover of a finite dimensional connected locally 6-large simplicial complex is contractible.

The proof of this theorem uses the projections onto closed combinatorial balls (compare [13, Section 8]). Restrictions of those projections to spheres

$$
\left.\pi_{B_{i}(Q, X)}\right|_{S_{i+1}(Q, X)}: S_{i+1}(Q, X) \rightarrow S_{i}(Q, X)
$$

have some properties that do not allow us to use them in order to define a reasonable boundary of a systolic complex. Thus in Section 3 we define, only for 7-systolic complexes, other maps between spheres.

The following lemma follows easily from the facts above and from [13, Corollary 1.5].

Lemma 2.6 Let $k \geq 6$, let $Y$ be a $k$-large simplicial complex and let $\sigma$ be a simplex of $X$. If $p: X \rightarrow Y$ is the universal cover of $Y$ and $m<\frac{k-1}{2}$ then for $i=0,1,2, \ldots, m$ the map $\left.p\right|_{B_{i}(\sigma, X)}: B_{i}(\sigma, X) \rightarrow B_{i}(p(\sigma), Y)$ is an isomorphism. 
We recall two results concerning systolic chamber complexes.

Lemma 2.7 [17, Lemma 4.1] Let $X$ be a systolic chamber complex of dimension $n \geq 1$ and $\tau$ its simplex. Then $S_{k}(\tau, X)$ is an $(n-1)$-dimensional chamber complex, for every $k \geq 1$.

Lemma 2.8 [17, Lemma 4.2] Let $X$ be a systolic chamber complex of dimension $n \geq 1$ and $\tau$ its simplex. Let $\sigma$ be an $(n-1)$-dimensional simplex of $S_{k}(\tau, X)$. Then there exists a vertex $v$ at a distance $k+1$ from $\tau$ such that $v * \sigma$ is a simplex of $X$.

\subsection{Strongly hereditarily aspherical compacta}

The notion of strongly hereditarily aspherical compacta was introduced by R J Daverman [5]. A compact metric space $Z$ is strongly hereditarily aspherical if it can be embedded in the Hilbert cube $Q$ in such a way that for each $\epsilon>0$ there exists an $\epsilon$-covering $\mathcal{U}$ of $Z$ by open subsets of $Q$, where the union of any subcollection of elements of $\mathcal{U}$ is aspherical.

To show, in Section 4, that the boundaries of 7-systolic groups are strongly hereditarily aspherical, we will use the following result.

Proposition 2.9 [5, Proposition 1] Suppose $\left\{L_{i}, \mu_{i}\right\}$ is an inverse sequence of finite polyhedra and PL maps, and suppose each $L_{i}$ is endowed with a fixed triangulation $T_{i}$ such that

(1) $\mu_{i}^{-1}$ (each subcomplex of $L_{i}$ ) is aspherical, and

(2) there exists a sequence $\left(a_{k}\right)_{k=1}^{\infty}$ of positive numbers, such that $\lim _{k \rightarrow \infty} a_{k}=0$, and $\operatorname{diam}\left(\mu_{i-k} \circ \ldots \circ \mu_{i-1}(\sigma)\right)<a_{k}$, for every simplex $\sigma \in T_{i}$.

Then the inverse limit $Z=\operatorname{inv} \lim \left\{L_{i}, \mu_{i}\right\}$ is strongly hereditarily aspherical.

It should be noticed that, by Daverman and Dranishnikov [6, Theorem 2.10], every strongly hereditarily aspherical compactum can be expressed as an inverse limit like the one above.

\section{7-systolic complexes}

In this section we study some properties of 7-systolic complexes. In particular we define and examine other (then in the general systolic case) contractions on spheres. 
This is crucial for Section 4. Then we study properties of some 7-systolic chamber complexes. Those results are important for Section 5.

Let $X$ be a 7 -systolic complex of dimension $n<\infty$. Let $Q$ be its convex subcomplex (see Sections 3 and 7 of [13] and compare Section 2.2). For a natural number $k$, we denote by $S_{k}$ the combinatorial sphere $S_{k}(Q, X)$ (compare Section 2.2) and we denote by $B_{k}$ the closed ball $B_{k}(Q, X)$.

Define a map $\pi_{Q, k}: S_{k}^{(0)} \rightarrow\left(S_{k-1}^{\prime}\right)^{(0)}$ by putting $\pi_{Q, k}(w)=b_{\tau}$, for every vertex $w$ of $S_{k}$, where $\tau=X_{w} \cap B_{k-1}$ is the projection of $w$ on $S_{k-1}$ and $b_{\tau} \in\left(S_{k-1}^{\prime}\right)^{(0)}$ is the barycenter of $\tau$.

Lemma 3.1 Let $v_{1}$ and $v_{2}$ be two vertices in $S_{k}$ belonging to the same simplex. Then $\pi_{Q, k}\left(v_{1}\right)$ and $\pi_{Q, k}\left(v_{2}\right)$ belong to the same simplex of $S_{k-1}^{\prime}$.

Proof Let $\tau=X_{\left\langle v_{1}, v_{2}\right\rangle} \cap B_{k-1}$ and $\tau_{i}=X_{v_{i}} \cap B_{k-1}$, for $i=1,2$. Then $\tau \subset \tau_{1} \cap \tau_{2}$. It is enough to show that $\tau_{1} \subset \tau_{2}$ or $\tau_{2} \subset \tau_{1}$.

We will show this arguing by contradiction. Suppose it is not true, ie there exist vertices $w_{i}$ such that $w_{i} \in \tau_{i} \backslash \tau_{j}$ for $\{i, j\}=\{1,2\}$. Let for $i=1,2, t_{i}$ be a vertex belonging to $X_{w_{i}} \cap B_{k-2} \cap X_{\tau}$.

Let us examine the closed path $\left(v_{1}, w_{1}, t_{1}, t_{2}, w_{2}, v_{2}, v_{1}\right)$ in $X^{(1)}$.

There are no diagonals of the form $\left\langle v_{i}, t_{j}\right\rangle$ since the distance between $v_{i}$ and $t_{j}$ is at least $2, i, j=1,2$.

There are no diagonals of the form $\left\langle v_{i}, w_{j}\right\rangle, i \neq j$. Since if, eg $\left\langle v_{1}, w_{2}\right\rangle$ is an edge in $X$ then $w_{2} \in X_{v_{1}} \cap B_{k-1}=\tau_{1}$.

There is no diagonal $\left\langle w_{1}, w_{2}\right\rangle$. If it exists, then the path $\left(v_{1}, w_{1}, w_{2}, v_{2}, v_{1}\right)$ is a closed simple path without diagonals of length 4 which contradicts 7-largeness of $X$. Similarly there are no diagonals $\left\langle w_{1}, t_{2}\right\rangle$ and $\left\langle w_{2}, t_{1}\right\rangle$.

Hence the path $\left(v_{1}, w_{1}, t_{1}, t_{2}, w_{2}, v_{2}, v_{1}\right)$ is a closed path of length at most six without diagonals. This contradicts 7 -largeness of $X$.

Using the above lemma we can extend $\pi_{Q, k}$ simplicially.

Definition 3.2 Define, for a natural number $k$, a continuous map between combinatorial spheres

$$
\pi_{Q, k}: S_{k}(Q, X) \rightarrow\left(S_{k-1}(Q, X)\right)^{\prime},
$$

given by the simplicial extension of the map

$$
{ }^{\pi_{Q, k}}: S_{k}(Q, X)^{(0)} \rightarrow\left(S_{k-1}(Q, X)^{\prime}\right)^{(0)} .
$$


Lemma 3.3 There exists a constant $C<1$, depending only on $n=\operatorname{dim}(X)$ such that for every $k, l \in\{1,2,3, \ldots\}$ with $l<k$ and for every two points $x, y \in S_{k}$ one has $\mathrm{d}_{S_{k-l-1}}\left(\pi_{Q, k-l} \circ \ldots \circ \pi_{Q, k-1} \circ \pi_{Q, k}(x), \pi_{Q, k-l} \circ \ldots \circ \pi_{Q, k-1} \circ \pi_{Q, k}(y)\right) \leq$ $C^{l+1} \mathrm{~d}_{S_{k}}(x, y)$.

Proof Let $D$ be the distance from a vertex to an opposite codimension one face in the regular $(n-1)$-simplex. Let $E$ be the diameter of a maximal simplex in the barycentric subdivision of the regular $(n-1)-$ simplex. Then for $C=\frac{E}{D}<1$ the lemma holds.

Lemma 3.4 For every subcomplex $L$ of $S_{k-1}$ the subcomplex $\pi_{Q, k}^{-1}(L)$ of $S_{k}$ is aspherical.

Proof We will show that $\pi_{Q, k}^{-1}(L)$ is a full subcomplex of $S_{k}$. Hence, by Proposition 2.1 , as a full subcomplex of 6-large complex it is 6-large and thus aspherical, by Theorem 2.5 .

Let vertices $v_{1}, v_{2}, \ldots, v_{l} \in\left(\pi_{Q, k}^{-1}(L)\right)^{(0)}$ span a simplex in $S_{k}$. Then, by Lemma 3.1, $\pi_{Q, k}\left(v_{1}\right), \ldots, \pi_{Q, k}\left(v_{l}\right)$ are vertices of a simplex of $S_{k-1}^{\prime}$ and they correspond to a chain of simplices $\tau_{1}, \ldots, \tau_{l}$ of $S_{k-1}$. One of them, say $\tau_{1}$, is the highest dimensional simplex among them and hence it contains all the simplices $\tau_{2}, \ldots, \tau_{l}$. This means that $\pi_{Q, k}^{-1}\left(\tau_{1}\right) \subset \pi_{Q, k}^{-1}(L)$ contains all points $v_{1}, \ldots, v_{l}$ and hence simplex spanned by them.

In the rest of this section we study some 7-large chamber complexes.

For a 7-large chamber complex $X$ we define, for a vertex $v$ of $X$, a condition $R(v, X)$ that will be crucial for Section 5 (compare the condition $R(v, X)$ defined in [17, Section 4]):

$R(v, X)$ iff (for all $\sigma \in X_{v}\left(X_{v} \backslash \stackrel{\circ}{B}_{2}\left(\sigma, X_{v}\right)\right.$ and $X_{v} \backslash \stackrel{\circ}{B}_{3}\left(\sigma, X_{v}\right)$ are connected))

The next lemma is an analogue of [17, Lemma 4.7], for 7-large complexes.

Lemma 3.5 Let $X$ be a 7-large chamber complex such that the link $X_{\kappa}$ is connected, for every simplex $\kappa$ of $X$ of codimension greater than one, and $X_{\sigma} \backslash \stackrel{\circ}{B}_{i}\left(\rho, X_{\sigma}\right)$, $i=2,3$ is connected for every codimension two simplex $\sigma$ of $X$ and every simplex $\rho$ of its link $X_{\sigma}$. Then for every vertex $v$ of $X$ condition $R(v, X)$ holds.

Proof We will proceed by induction on $n=\operatorname{dim}(X)$.

For $n=2$ the assertion is clear since codimension two simplices are just vertices. 
Assume we proved the lemma for $n \leq k$. Let $\operatorname{dim}(X)=k+1$. Take a vertex $v$ of $X$ and consider its link $X_{v}$. It has dimension $k$. Moreover for every codimension $l$ simplex $\sigma$ of $X_{v}$ the simplex $\sigma * v$ is of codimension $l$ in $X$ and $X_{\sigma * v}=\left(X_{v}\right)_{\sigma}$. Thus $X_{v}$ satisfies hypotheses of the lemma. Hence by the induction assumptions, for every vertex $w$ of $X_{v}$ condition $R\left(w, X_{v}\right)$ holds.

$X_{v}$ is 7-large as a full subcomplex of $X$ (Proposition 2.1). Thus the universal cover $\widetilde{X}_{v}$ of $X_{v}$ is 7-systolic. Let $p: \widetilde{X}_{v} \rightarrow X_{v}$ be a covering map.

Take a simplex $\omega$ of $X_{v}$. Let $\kappa$ be a simplex of $\widetilde{X}_{v}$ such that $p(\kappa)=\omega$. Since $S_{0}\left(\kappa, \widetilde{X}_{v}\right)$ is connected we have, by [17, Lemma 4.5] (or by Corollary 5.4), that $S_{1}\left(\kappa, \widetilde{X_{v}}\right), S_{2}\left(\kappa, \widetilde{X_{v}}\right)$ and $S_{3}\left(\kappa, \widetilde{X_{v}}\right)$ are connected. By Lemma 2.6,

$$
p^{\prime}=\left.p\right|_{B_{2}\left(\kappa, \widetilde{X_{v}}\right)}: B_{2}\left(\kappa, \widetilde{X_{v}}\right) \rightarrow B_{2}\left(\omega, X_{v}\right)
$$

is an isomorphism. Observe that $p\left(B_{3}\left(\kappa, \widetilde{X_{v}}\right)\right) \subset B_{3}\left(\omega, X_{v}\right)$. We want to show that $p\left(B_{3}\left(\kappa, \widetilde{X_{v}}\right)\right)=B_{3}\left(\omega, X_{v}\right)$. Let $z$ be a vertex in $B_{3}\left(\omega, X_{v}\right) \backslash B_{2}\left(\omega, X_{v}\right)$ and let $u \in B_{2}\left(\omega, X_{v}\right)$ be a vertex connected by an edge with $z$. Then, by Lemma 2.6,

$$
p^{\prime \prime}=\left.p\right|_{B_{1}\left(\left(p^{\prime}\right)^{-1}(u), \widetilde{X}_{v}\right)}: B_{1}\left(\left(p^{\prime}\right)^{-1}(u), \widetilde{X}_{v}\right) \rightarrow B_{1}\left(u, X_{v}\right)
$$

is an isomorphism and $\left(p^{\prime \prime}\right)^{-1}(z) \in B_{3}\left(\kappa, \widetilde{X}_{v}\right)$. Hence $z \in p\left(B_{3}\left(\kappa, \widetilde{X}_{v}\right)\right)$ and the image $p\left(B_{3}\left(\kappa, \widetilde{X_{v}}\right)\right)=B_{3}\left(\omega, X_{v}\right)$. Now we claim that $S_{3}\left(\omega, X_{v}\right)=p\left(S_{3}\left(\kappa, \widetilde{X_{v}}\right)\right)$ and hence is connected. Observe that $S_{3}\left(\omega, X_{v}\right) \subset p\left(S_{3}\left(\kappa, \widetilde{X}_{v}\right)\right)$. Suppose $S_{3}\left(\omega, X_{v}\right) \neq$ $p\left(S_{3}\left(\kappa, \widetilde{X}_{v}\right)\right)$. Let $w_{1} \in S_{3}\left(\kappa, \widetilde{X}_{v}\right)$ be a vertex such that $p\left(w_{1}\right) \in B_{2}\left(\omega, X_{v}\right)$. The vertex $w_{2}=\left(p^{\prime}\right)^{-1}\left(p\left(w_{1}\right)\right)$ belongs to $B_{2}\left(\kappa, \widetilde{X}_{v}\right)$ and $p\left(w_{1}\right)=p\left(w_{2}\right)$. But then the distance between $w_{1}$ and $w_{2}$ in $\widetilde{X}_{v}{ }^{(1)}$ is less than 7 and we can find homotopically nontrivial closed path of length less than 7 in $X_{v}$. This contradicts 7-largeness of $X_{v}$, by [13, Corollary 1.5]. Thus we have shown that $S_{3}\left(\omega, X_{v}\right)=p\left(S_{3}\left(\kappa, \widetilde{X}_{v}\right)\right)$ is connected.

Take two vertices $t$ and $s$ of $X_{v} \backslash \stackrel{\circ}{B}_{2}\left(\omega, X_{v}\right)$ (or of $X_{v} \backslash \stackrel{\circ}{B}_{3}\left(\omega, X_{v}\right)$ ). Since, by assumptions, $X_{v}$ is connected there exists a path in $\left(X_{v}\right)^{(1)}$ joining them. If this path misses $\stackrel{\circ}{B}_{2}\left(\omega, X_{v}\right)$ (respectively $\left.\stackrel{\circ}{B}_{3}\left(\omega, X_{v}\right)\right)$ it joins these vertices in $X_{v} \backslash \stackrel{\circ}{B}_{2}\left(\omega, X_{v}\right)$ (respectively in $X_{v} \backslash \stackrel{\circ}{B}_{3}\left(\omega, X_{v}\right)$ ). If not we can replace it, by connectedness of $S_{2}\left(\omega, X_{v}\right)$ (respectively $S_{3}\left(\omega, X_{v}\right)$ ), by a path intersecting $S_{2}\left(\omega, X_{v}\right)$ (respectively $S_{3}\left(\omega, X_{v}\right)$ ) and also lying in $X_{v} \backslash \stackrel{\circ}{B}_{2}\left(\omega, X_{v}\right)$ (respectively in $X_{v} \backslash \stackrel{\circ}{B}_{3}\left(\omega, X_{v}\right)$ ). Hence we get the conclusion.

Corollary 3.6 Let $X$ be a normal 7-systolic pseudomanifold. Then condition $R(v, X)$ holds for every vertex $v$ of $X$. 
Proof One dimensional link in a normal pseudomanifold is a circle. Hence it satisfies assumptions of the preceding corollary.

\section{Gromov boundary}

The aim of this section is to prove that the ideal boundary of a 7-systolic group (such groups are word hyperbolic by Theorem 2.2) is a strongly hereditarily aspherical compactum. To prove this we first show that such a boundary can be described as an inverse limit of combinatorial spheres in the complex on which the group acts geometrically.

Throughout this section $X$ denotes a locally finite 7-systolic complex of dimension $n<\infty$. We fix a vertex $v$ of $X$. For a natural number $k$, we denote by $S_{k}$ the combinatorial sphere $S_{k}(v, X)$ and we denote by $B_{k}$ the closed ball $B_{k}(v, X)$. We denote by $\pi_{k}$ the projection $\pi_{\{v\}, k}: S_{k} \rightarrow S_{k-1}$ (see Section 3).

Lemma 4.1 $\delta X=\operatorname{inv} \lim \left\{S_{k}, \pi_{k}\right\}$ is homeomorphic to $\partial X$, the Gromov boundary of $X$.

Proof We use the set of equivalence classes of geodesic rays in $X^{(1)}$ propagating from a given vertex $v$, as a definition of the Gromov boundary of $X$-for details see eg Bridson and Haefliger [3, Chapter III.3].

Compactness of both $\delta X$ and $\partial X$ follows from the fact that the balls in $X^{(0)}$ are finite.

First, we construct a bijection $F: \delta X \rightarrow \partial X$. Let $x=\left(v, x_{1}, x_{2}, \ldots\right) \in \delta X$. Note that $x_{k} \in S_{k}$ for $k=1,2, \ldots$. For arbitrary $k$, choose a maximal simplex $\sigma_{k}$ of $S_{k}$ containing $x_{k}$. If we take a vertex $u$ of $\sigma_{k}$ then $X_{\sigma_{k}} \cap B_{k-1} \subset X_{u} \cap B_{k-1}$ and hence there exists a vertex $w$ of $\sigma_{k-1}$ connected by an edge with $u$. Hence for any $k$ we can construct a sequence $\left(v=v_{0}^{k}, v_{1}^{k}, v_{2}^{k}, \ldots, v_{k}^{k}\right)$ of vertices of $X$ such that $v_{i}^{k} \in \sigma_{i}$ and $v_{k}^{i-1}$ is connected by an edge with $v_{k}^{i}$ for $i=1,2, \ldots, k$. Since a path $c_{k}=\left(v_{0}^{k}, \ldots, v_{k}^{k}\right)$ in $X^{(1)}$ has length $k$ and joins $v$ and $v_{k}^{k}$ lying at a distance $k$ it is a geodesic segment starting at $v$. Since balls in $X^{(0)}$ are finite, we can, by the diagonal argument, extract from $\left(c_{k}\right)_{k=1}^{\infty}$ a geodesic ray $c=\left(v, v_{1}, v_{2}, \ldots\right)$, such that $v_{k}$ is a vertex of $\sigma_{k}$, for every $k=1,2,3, \ldots$. The equivalence class of $c$ within $\partial X$ is by definition $F(x)$. Observe that it is independent of choosing $c$ as above, since all of them lie at distance at most one from the sequence $x$.

We show now that $F$ is injective. Let $x=\left(v, x_{1}, x_{2}, \ldots\right)$ and $y=\left(v, y_{1}, y_{2}, \ldots\right)$ be two elements of $\delta X$ with $F(x)=F(y)$. Let the geodesic rays $c=\left(v, v_{1}, v_{2}, \ldots\right)$ and $d=\left(v, w_{1}, w_{2}, \ldots\right)$ in $X^{(1)}$ representing, respectively, $F(x)$ and $F(y)$ be constructed 
as above. Then there exists a constant $D>0$ such that for every $k=1,2,3, \ldots$ we have $d_{S_{k}}\left(v_{k}, w_{k}\right) \leq D$. Fix $k$. It is enough to show that $d_{S_{k}}\left(x_{k}, y_{k}\right) \leq \epsilon$ for every $\epsilon>0$. Choose $\epsilon>0$. Take $l \in \mathbf{N}$ such that $l \geq \log _{C} \frac{\epsilon}{D+2}$, where $C<1$ is the constant from the proof of Lemma 3.3. By construction $d_{S_{k+l}}\left(x_{k+l}, y_{k+l}\right) \leq D+2$ and thus by Lemma 3.3,

$$
\begin{aligned}
& d_{S_{k}}\left(x_{k}, y_{k}\right) \\
& \quad=d_{S_{k}}\left(\pi_{k+1} \circ \ldots \circ \pi_{k+l-1} \circ \pi_{k+l}\left(x_{k+l}\right), \pi_{k+1} \circ \ldots \circ \pi_{k+l-1} \circ \pi_{k+l}\left(y_{k+l}\right)\right) \\
& \quad \leq C^{l} d_{S_{k+l}}\left(x_{k+l}, y_{k+l}\right) \leq \epsilon .
\end{aligned}
$$

Now we show $F$ is onto. Take a geodesic ray $c=\left(v, v_{1}, v_{2}, \ldots\right), v_{i} \in X^{(0)}$. Observe that $v_{k} \in S_{k}$ for $k=2,3,4, \ldots$. Consider a sequence $\left(\pi_{2} \circ \pi_{3} \circ \ldots \circ \pi_{k}\left(v_{k}\right)\right)_{k=2}^{\infty}$ of points in $S_{1}$. By compactness of spheres there is a subsequence $\left(v_{a^{1}(1)}, v_{a^{1}(2)}, \ldots\right)$ of the sequence $\left(v_{2}, v_{3}, \ldots\right)$ such that $\left(\pi_{2} \circ \pi_{3} \circ \ldots \circ \pi_{a^{1}(k)}\left(v_{a^{1}(k)}\right)\right)_{k=2}^{\infty}$ converges. Let $x_{1} \in S_{1}$ be the limit of this subsequence. Now given a subsequence (of $\left(v, v_{1}, v_{2}, \ldots\right)$ ) $\left(v_{a^{l}(1)}, v_{a^{l}(2)}, \ldots\right), l>1$ we find a subsequence $\left(v_{a^{l+1}(1)}, v_{a^{l+1}(2)}, \ldots\right), a^{l+1}(i)>l$ such that $\left.\pi_{l+2} \circ \ldots \circ \pi_{a^{l+1}(k)}\left(v_{a^{l+1}(k)}\right)\right)_{k=l+1}^{\infty}$ tends to $x_{l+1} \in S_{l+1}$. By construction $\pi_{k}\left(x_{k}\right)=x_{k-1}$, for $k>1$ and $\pi_{1}\left(x_{1}\right)=v$. Hence $x=\left(v, x_{1}, x_{2}, \ldots\right) \in \delta X$. Moreover since $v_{k}$ and $v_{k+1}$ belong to a common simplex for every $k=2,3,4 \ldots$ we get, by definition of $\pi_{k}$, that $d\left(v_{k}, \pi_{k+1}\left(v_{k+1}\right)\right) \leq E$, for $E<1$ being the constant from the proof of Lemma 3.3. Then for every natural number $l$ we have the inequality $d\left(v_{k}, \pi_{k+1} \circ \pi_{k+2} \circ \ldots \circ \pi_{k+l}\left(v_{k+l}\right)\right) \leq \sum_{i=1}^{\infty} E^{i}<\infty$. Thus $d\left(v_{k}, x_{k}\right)<\sum_{i=1}^{\infty} E^{i}<$ $\infty$, which implies $c$ represents the equivalence class of $F(x)$.

Finally we argue $F$ is continuous and hence as a continuous bijection defined on a compact space it is a homeomorphism.

Given $x=\left(v, x_{1}, x_{2}, \ldots\right) \in \delta X$ and a sequence $\left(x^{i}\right)_{i=1}^{\infty} \subset \delta X, x^{i}=\left(v, x_{1}^{i}, \ldots\right)$ converging to $x$, fix geodesic rays $c=\left(v, v_{1}, v_{2}, \ldots\right)$ and $c_{i}=\left(v, v_{1}^{i}, v_{2}^{i}, \ldots\right), i=1,2,3, \ldots$ representing, respectively, $F(x)$ and $F\left(x^{i}\right), i=1,2,3, \ldots$, and constructed as when we defined $F$. To prove $F$ is continuous at $x$ it is enough to show that for every natural number $N$ there exists $M>0$ such that for every natural number $i>M$ we have $d_{S_{N}}\left(v_{N}, v_{N}^{i}\right)<3$. By definition of the topology of an inverse limit there exists $M>0$ such that for every natural number $i>M$ one has $d_{S_{N}}\left(x_{N}, x_{N}^{i}\right)<1$ and hence $d_{S_{N}}\left(v_{N}, v_{N}^{i}\right) \leq d_{S_{N}}\left(v_{N}, x_{N}\right)+d_{S_{N}}\left(x_{N}, x_{N}^{i}\right)+d_{S_{N}}\left(x_{N}^{i}, v_{N}^{i}\right)<3$.

Now we state and prove the following main theorem.

Theorem 4.2 The ideal boundary of a 7-systolic group is a strongly hereditarily aspherical compactum. 
Proof A 7-systolic group $G$ acts, by definition, geometrically on a locally finite 7 -systolic complex $X$ of finite dimension. Then the ideal boundary $\partial G$ of $G$ is homeomorphic to $\partial X$.

We apply Proposition 2.9 to the inverse system $\left\{L_{i}, \mu_{i}\right\}=\left\{S_{i}, \pi_{i+1}\right\}$. By Lemma 3.4 the condition 1) of Proposition 2.9 is fulfilled, and by Lemma 3.3 we get condition 2) of the proposition. Hence $\partial G=\partial X=\delta X=\operatorname{inv} \lim \left\{S_{i}, \pi_{i+1}\right\}$ is a strongly hereditarily aspherical compactum.

Remarks 4.3 (1) A simple argument shows that every compact metrizable space can be homeomorphic to the ideal boundary of some hyperbolic space (even more-of some $C A T(-1)$ space). The question of which topological spaces can occur as boundaries of hyperbolic groups (compare Benakli and Kapovich [15, Chapter 17]) is more difficult. It is answered somehow only in dimensions (of the boundary) 0 and 1 (cf Kapovich and Kleiner [16]). For higher dimensions the following homeomorphism types of the boundaries of hyperbolic groups were known: spheres, Pontryagin surfaces $\Pi_{p}$ for $p$ being a prime number, two-dimensional universal Menger compactum $\mu_{2}^{5}$ (compare [15, Chapter 17]), three-dimensional universal Menger compactum $\mu_{3}^{7}$ (cf Dymara and Osajda [7]), Pontryagin spheres and three-dimensional trees of manifolds (cf Przytycki and Świątkowski [18]).

By Theorem 2.2, 7-systolic groups are hyperbolic and, by [13, Corollary 19.3], for each natural number $n$, there exists a hyperbolic group of cohomological dimension $n$. Hence, by Theorem 4.2, and by Bestvina and Mess [1, Corollary 1.4], strongly hereditarily aspherical compacta of all dimensions can occur as boundaries of hyperbolic groups.

Moreover, in [13] examples of 7-systolic groups acting on pseudomanifolds of arbitrary large dimension are constructed. Thus, by Corollaries 5.7 and 5.9, those group are, in a sense, indecomposable and their boundaries are connected, locally connected and without local cut points (compare Section 5).

(2) Zawiślak [20] has shown that the boundary of a 7-systolic orientable normal pseudomanifold of dimension 3 is the Pontryagin sphere (cf Jakobsche [11]). Such pseudomanifolds are constructed in [13].

The Pontryagin sphere is the inverse limit of an inverse system $\left\{X_{i}, p_{i}\right\}_{i=1}^{\infty}$ defined as follows. Let $X_{1}=S^{2}$ be a triangulated two-sphere. Let $\mathcal{T}$ be a given triangulation of the two torus. Assume $X_{i}, p_{j}$ are defined for $i \leq k$ and $j \leq k-1$. Let $X_{k}$ be a surface and $\mathcal{T}_{k}$ its triangulation. $X_{k+1}$ is a connected sum of $X_{k}$ and a set of disjoint tori $T_{\sigma}$-one for every 2-simplex $\sigma$ of $\mathcal{T}_{k}$ - carrying the triangulation $\mathcal{T}$. Every $T_{\sigma}$ is glued to $X_{k}$ by identifying $\partial \sigma$ and the boundary of some 2-simplex $\sigma^{\prime}$ of triangulation 
of $T_{\sigma}$. Then $X_{k+1}$ carries an induced triangulation and we define a triangulation $\mathcal{T}_{k+1}$ of $X_{k+1}$ as a subdivision of this natural triangulation. The map $p_{k}: X_{k+1} \rightarrow X_{k}$ is defined by the conditions: $p_{k}\left(T_{\sigma} \backslash \sigma^{\prime}\right)=$ int $\sigma$ and $\left.p_{k}\right|_{\partial \sigma}=I d_{\partial \sigma}$ for every 2-simplex $\sigma$ of $\mathcal{T}_{k}$.

(3) For a polytopal complex $Y$ its face complex $\Phi(Y)$ is a simplicial complex defined as follows. The vertex set of $\Phi(Y)$ is the set of cells of $Y$ and the vertices of $\Phi(Y)$ span a simplex if the cells of $Y$ corresponding to those vertices are contained in a common cell of $Y$. It can be shown (compare [12]) that if $Y$ is a simply connected simple (ie all links are simplicial complexes) polytopal complex with 7-large links then $\Phi(Y)$ is 7 -systolic. Thus the ideal boundary of such a complex $Y$ is strongly hereditarily aspherical.

Question Is the ideal boundary of a hyperbolic systolic group strongly hereditarily aspherical?

\section{Splittings}

The aim of this section is to study further properties of boundaries of 7-systolic complexes in some special cases. As a consequence we get results concerning splittings of groups acting on such complexes.

Throughout this section $X$ denotes a locally finite 7-systolic chamber complex of dimension $n<\infty$. We fix a vertex $v$ of $X$. For a natural number $k$, we denote by $S_{k}$ the combinatorial sphere $S_{k}(v, X)$ and we denote by $B_{k}$ the closed ball $B_{k}(v, X)$. We denote by $\pi_{k}$ the projection $\pi_{\{v\}, k}: S_{k} \rightarrow S_{k-1}$ (see Section 3).

Lemma 5.1 Let $Y$ be a 7-large $n$-dimensional chamber complex, $\sigma$ one of its simplices and $\tau$ an $(n-1)$-simplex of $S_{2}(\sigma, Y)$. Then there exists a vertex $v \in$ $Y \backslash B_{2}(\sigma, Y)$ such that $v * \tau$ is a simplex of $Y$.

Proof By Lemma 2.8, if we consider the universal cover $p: \tilde{Y} \rightarrow Y$ and $\widetilde{\sigma} \in p^{-1}(\sigma)$, $\tilde{\tau} \in p^{-1}(\tau) \cap S_{2}(\tilde{\sigma}, \tilde{Y})$, then there exists a vertex $\tilde{v}$ of $\tilde{Y}$ such that $\tilde{v} \in \tilde{Y} \backslash B_{2}(\widetilde{\sigma}, \tilde{Y})$ and $\tilde{v} * \tilde{\tau}$ is a simplex of $\tilde{Y}$. Consider $v=p(\widetilde{v})$. Clearly $v * \tau$ is a simplex of $Y$.

Assume $v \in B_{2}(\sigma, Y)$. Then there exists a simplex $\widetilde{\sigma_{1}} \in p^{-1}(\sigma)$ distinct from $\widetilde{\sigma}$ such that $\tilde{v} \in B_{2}\left(\widetilde{\sigma_{1}}, \tilde{Y}\right)$. Since $\widetilde{v} \in B_{3}(\widetilde{\sigma}, \tilde{Y})$ we can then choose vertices $s \in \widetilde{\sigma}$ and $t \in \widetilde{\sigma_{1}}$ with $p(s)=p(t)$ and a path of length at most 6 joining $s$ and $t$. But this contradicts 7 -largeness of $Y$. Thus $v \in Y \backslash B_{2}(\sigma, Y)$. 
Lemma 5.2 The map $\pi_{k}: S_{k} \rightarrow S_{k-1}$ is onto.

Proof Let $z$ be a given point in $S_{k-1}$. We will show that there exists a point $w \in S_{k}$ satisfying $\pi_{k}(w)=z$.

Case $1 z$ is a barycenter of a simplex $\tau$ of $S_{k-1}$.

If $\operatorname{dim}(\tau)=n-1$ then by Lemma 2.8 there exists a vertex $w \in S_{k}$ such that $w * \tau$ is a simplex of $X$ and hence $\pi_{k}(w)=z$.

Now, let $\operatorname{dim}(\tau)=m<n-1$. Since, by Lemma 2.7, $S_{k-1}$ is a chamber complex of dimension $n-1$, there exists an $(n-1)-\operatorname{simplex} \rho$ of $S_{k-1}$ containing $\tau$. Then, again by Lemma 2.8 , there exists a vertex $w^{\prime} \in S_{k}$ spanning a simplex with $\rho$. Clearly $w^{\prime} \in S_{k} \cap S_{2}\left(\delta, X_{\tau}\right)$, where $\delta=X_{\tau} \cap S_{k-2}$. Since $X_{\tau}$ is a 7-large $(n-m-1)$ dimensional chamber complex and, (again by Lemma 2.7) $S_{2}\left(\delta, X_{\tau}\right)$ is an $(n-m-2)-$ dimensional chamber complex (nonempty), we get, by Lemma 5.1, that there exists a vertex $w \in X_{\tau} \backslash B_{2}\left(\delta, X_{\tau}\right)$. It follows that $\pi_{k}(w)=z$.

Case $2 z$ belongs to an interior of an $m$-simplex $\tau$ of $S_{k-1}^{\prime}$.

Then $\tau=\left\langle a_{0}, a_{1}, \ldots, a_{m}\right\rangle$ where $a_{i}$ is the barycenter of an $i$-simplex $\tau_{i}$ of $S_{k-1}$. By Case 1 there exists a vertex $a_{m}^{\prime} \in S_{k}$ such that $\pi_{k}\left(a_{m}^{\prime}\right)=a_{m}$. Then we have $a_{m}^{\prime} \in S_{2}\left(X_{\tau_{m-1}} \cap B_{k-2}, X_{\tau_{m-1}}\right)$ and, using Lemma 5.1 for $X_{\tau_{m-1}}$, there exists a vertex $a_{m-1}^{\prime} \in X_{\tau_{m-1}} \backslash B_{2}\left(X_{\tau_{m-1}} \cap B_{k-2}, X_{\tau_{m-1}}\right)$ connected to $a_{m}^{\prime}$ by an edge. Note that $\pi_{k}\left(a_{m-1}^{\prime}\right)=a_{m-1}$ and that $\left\langle a_{m}^{\prime}, a_{m-1}^{\prime}\right\rangle \subset S_{2}\left(X_{\tau_{m-2}} \cap B_{k-2}, X_{\tau_{m-2}}\right)$. Assume we found vertices $a_{m}^{\prime}, a_{m-1}^{\prime}, \ldots, a_{l}^{\prime}, l>0$ spanning a simplex in $S_{2}\left(X_{\tau_{l-1}} \cap\right.$ $\left.B_{k-2}, X_{\tau_{l-1}}\right)$, such that $\pi_{k}\left(a_{i}^{\prime}\right)=a_{i}$. Then we can find a vertex $a_{l-1}^{\prime} \in X_{\tau_{l-1}} \backslash$ $B_{2}\left(X_{\tau_{l-1}} \cap B_{k-2}, X_{\tau_{l-1}}\right)$ spanning together with $\left\langle a_{m}^{\prime}, a_{m-1}^{\prime}, \ldots, a_{l}^{\prime}\right\rangle$ a simplex in $X$. Hence we can find points $a_{m}^{\prime}, a_{m-1}^{\prime}, \ldots, a_{0}^{\prime} \in S_{k}$ spanning a simplex in $X$ and satisfying $\pi_{k}\left(a_{i}^{\prime}\right)=a_{i}$. Then if $z=\sum_{i=0}^{m} \lambda_{i} a_{i}$ for $\lambda_{i}>0$ such that $\sum_{i=0}^{m} \lambda_{i}=1$, we have $\pi_{k}\left(\sum_{i=0}^{m} \lambda_{i} a_{i}^{\prime}\right)=z$.

Lemma 5.3 Let the condition $R(w, X)$ hold for every vertex $w$ of $X$. Then $\pi_{k}^{-1}(\rho)$ is connected for every simplex $\rho$ of $S_{k-1}$ and for every $k \geq 2$.

Proof If $\rho$ is a vertex then its preimage by the map $\pi_{k}: S_{k} \rightarrow S_{k-1}, \pi_{k}^{-1}(\rho)=$ span $\left\{\right.$ vertices in $\left.X_{\rho} \backslash B_{2}\left(X_{\rho} \cap B_{k-2}, X_{\rho}\right)\right\}$ is nonempty, by Lemma 5.2 and it is connected by $R(\tau, X)$.

By surjectivity of $\pi_{k}$ it is now enough to show the following. Let $\tau=\left\langle a_{0}, a_{1}, \ldots, a_{m}\right\rangle$ be a simplex of $S_{k-1}^{\prime}$ such that $a_{i}$ is the barycenter of an $i$-simplex of $S_{k-1}$. Then every point $p \in \pi_{k}^{-1}(\rho)$ can be connected to $\pi_{k}^{-1}\left(a_{0}\right)$ by a path in $\pi_{k}^{-1}(\tau)$. 
The proof goes via induction on $m$.

For $m=0$ it is trivial.

Let $m>0$. Wlog we can assume that $\pi_{k}(p)=\sum_{i=0}^{m} \lambda_{i} a_{i}$ with $\lambda_{m}>0$ and $\sum_{i=0}^{m} \lambda_{i}=$ $1, \lambda_{i} \geq 0$. Let $I \subset\left\{i \mid \lambda_{i} \neq 0\right\}$ By definition of $\pi_{k}$ there exist $\left(a_{j}^{\prime}\right)_{j \in I}$ such that $\pi_{k}\left(a_{j}^{\prime}\right)=a_{j}, \sum_{j \in I} \lambda_{j} a_{j}^{\prime}=p$. Then the span of $\left(a_{j}^{\prime}\right)_{j \in I}$ is contained in $\pi_{k}^{-1}(\tau)$ and hence we can connect $p$ to $a_{m}^{\prime}$ inside $\pi_{k}^{-1}(\tau)$. Following Case 2 in the proof of Lemma 5.2 we can then find a vertex $a_{m-1}^{\prime \prime} \in S_{k}$ such that $\pi_{k}\left(a_{m-1}^{\prime \prime}\right)=a_{m-1}$ and $a_{m}^{\prime}$ and $a_{m-1}^{\prime \prime}$ span an edge. Then $\left\langle a_{m}^{\prime}, a_{m-1}^{\prime \prime}\right\rangle \subset \pi_{k}^{-1}(\tau)$ and, by induction assumptions, we can connect $p$ to $\pi_{k}^{-1}\left(a_{0}\right)$ by a path in $\pi_{k}^{-1}(\tau)$.

Corollary 5.4 Let the condition $R(w, X)$ hold $X$ for every vertex $w$ of $X$. Then for every $k \geq 2$ and for any connected subcomplex $K$ of $S_{k-1}$ its preimage $\pi_{k}^{-1}(K)$ is connected.

Theorem 5.5 Let $X$ be a finitely dimensional locally finite 7-systolic chamber complex such that the condition $R(w, X)$ holds for every vertex $w$ of $X$. Then the ideal boundary $\partial X$ of $X$ is connected.

Proof Observe that $S_{1}=X_{v}$ and thus it is connected by $R(v, X)$. By Corollary 5.4 if $S_{k-1}$ is connected then $S_{k}$ is connected too. Hence $\partial X$ as an inverse limit of continua is a continuum.

Theorem 5.6 Let $X$ be a locally finite 7-systolic chamber complex of finite dimension $n \geq 3$. Assume that the link $X_{\kappa}$ is connected, for every simplex $\kappa$ of $X$ of codimension greater then one, and $X_{\sigma} \backslash \stackrel{\circ}{B}_{i}\left(\rho, X_{\sigma}\right), i=2,3$ is connected for every codimension two simplex $\sigma$ of $X$ and every simplex $\rho$ of its link $X_{\sigma}$. Then the ideal boundary $\partial X$ of $X$ is connected and has no local cut points.

Proof Connectedness of the boundary follows from Lemma 3.5 and Theorem 5.5.

Now we show there are no local cut points in $\partial X$. If a point $x \in \partial X$ disconnects an open connected set $U \subset \partial X$ then it disconnects every open connected $V \subset U$. Hence it disconnects every connected subset $W \subset U$ whose interior contains $x$. Thus, to prove the Lemma, it is enough to show that for a given point $x \in \partial X$ and its open neighborhood $U$ there exists a connected set $W$ with $x \in$ int $W \subset W \subset U$ such that $W \backslash\{x\}$ is connected.

Let us define, for a natural number $k$, a map $\pi_{k}^{\infty}: \partial X \rightarrow S_{k}$ as a projection from the inverse limit $\partial X$ to the element $S_{k}$ of the inverse system $\left\{S_{i}, \pi_{i}\right\}$. By the definition 
of the topology on $\partial X$ we can find a natural number $k$ large enough so that if $\tau$ is a simplex of $S_{k}$ containing $\pi_{k}^{\infty}(x)$ then $W=\left(\pi_{k}^{\infty}\right)^{-1}\left(B_{2}\left(\tau, S_{k}\right)\right) \subset U$. We claim $W$ is as desired.

First observe that $\left(\pi_{k}^{\infty}\right)^{-1}\left(\stackrel{\circ}{B}_{2}\left(\tau, S_{k}\right)\right) \subset W$ is open and contains $x$. Moreover $B_{2}\left(\tau, S_{k}\right)$ is a connected subcomplex of $S_{k}$ and hence, by Corollary 5.4 the inverse system

$$
\left\{W_{l}=\pi_{l}^{-1}\left(\ldots\left(\pi_{k+1}^{-1}\left(B_{2}\left(\tau, S_{k}\right)\right)\right) \ldots\right),\left.\pi_{l}\right|_{W_{l}}\right\}_{l=k+1}^{\infty}
$$

consists of continua and its inverse limit $W$ is a continuum.

Now we show that every two points $y, z \in W \backslash\{x\}$ are connected by a continuum within $W \backslash\{x\}$. Again by the definition of the topology on $\partial X$ we can find $m$ big enough such that there exists a vertex $w \in S_{m}$ such that $\pi_{m}^{\infty}(x) \in \stackrel{\circ}{B}_{1}\left(w, S_{m}\right)$ and $y, z \notin \stackrel{\circ}{B}_{1}\left(w, S_{m}\right)$. Since $\pi_{m}^{\infty}(W)=\pi_{m}^{-1}\left(\ldots\left(\pi_{k+2}^{-1}\left(\pi_{k+1}^{-1}\left(B_{2}\left(\tau, S_{k}\right)\right)\right)\right) \ldots\right)$ is a connected subcomplex of $S_{m}$ then, if $S_{1}\left(w, S_{m}\right)=\left(S_{m}\right)_{w}$ is connected, we can find a continuum $K \in \pi_{m}^{\infty}(W) \backslash \stackrel{\circ}{B}_{1}\left(w, S_{m}\right)$ connecting $y$ and $z$. Then $\left(\pi_{m}^{\infty}\right)^{-1}(K)$ is a continuum (as an inverse limit of continua) in $W$ missing $x$ and containing $y$ and $z$.

Thus to finish the proof we have to show that $S_{1}\left(w, S_{m}\right)=\left(S_{m}\right)_{w}$ is connected. Observe that for every simplex $\sigma$ of $X_{w}$ the link of $\sigma$ in $X_{w}$ is the link of $\sigma * w$ in $X$. Hence (compare Lemma 3.5 and its proof) the link $X_{w}$ is a 7-large chamber complex such that the condition $R\left(z, X_{w}\right)$ holds for every vertex $z$, provided $X_{w}$ has dimension above two. Let $\rho=X_{w} \cap S_{m-1}$. Then, by Lemma 2.4, we get $S_{1}\left(w, S_{m}\right)=\left(S_{m}\right)_{w}=X_{w} \cap S_{m}=S_{1}\left(\rho, X_{w}\right)$. Since $\partial \rho$ is connected and balls of small radii in 6-large complexes are isomorphic with the ones in its universal covers (cf Lemma 2.6) we get, by Corollary 5.4 that $S_{1}\left(\rho, X_{w}\right)$ is connected.

Corollary 5.7 Let $X$ be a locally finite normal 7-systolic pseudomanifold of finite dimension at least 3 . Then its ideal boundary $\partial X$ is connected and has no local cut points.

Proof One-dimensional links in normal manifolds are circles.

Theorem 5.8 Let $G$ be a group acting geometrically by automorphisms on a 7systolic chamber complex $X$ of dimension $n \geq 3$. Assume that the link $X_{\kappa}$ is connected, for every simplex $\kappa$ of $X$ of codimension greater then one, and $X_{\sigma} \backslash \stackrel{\circ}{B}_{i}\left(\rho, X_{\sigma}\right), i=2,3$ is connected for every codimension two simplex $\sigma$ of $X$ and every simplex $\rho$ of its link $X_{\sigma}$. Then $G$ does not split essentially, as an amalgamated product or as an $H N N$-extension, over a finite nor two-ended group. 
Proof This follows from Theorem 5.6.

By Stallings' theorem [19], $G$ does not split over a finite group—compare also Gromov [9, remarks after Proposition 3.2.A], Coornaert-Delzant-Papadopoulos [4, Exercise 4) in Chapter 2] and Ghys and de la Harpe [8, Proposition 17 in Chapter 7.5].

By Bowditch [2, Theorem 6.2], $G$ does not split essentially over a two-ended group.

Corollary 5.9 A group acting geometrically by automorphisms on a locally finite normal 7-systolic pseudomanifold of dimension at least 3 does not split essentially over a finite or two-ended group as an amalgamated product or an $\mathrm{HNN}$-extension.

Remarks 5.10 (1) A systolic group acting on a 7-systolic pseudomanifold of dimension at least 3 can split over a surface group (this remark is due to J Świątkowski). To see this take two isomorphic closed 3-dimensional 7-large pseudomanifolds with links of vertices being closed surfaces (such spaces exist by Januszkiewicz and Świątkowski [13, Corollary 19.3 (1) and its proof]). Consider complement of an open residue of a given vertex in each of them. The link of the vertex is a convex subcomplex of the complement and hence the union of both complements along that links is 7-large. Thus the fundamental group of the sum splits over the fundamental group of a link which is a surface.

(2) As noticed in [17, Section 5] most of the examples of systolic groups-except automorphism groups of complexes of dimension at most two-constructed in [13] as fundamental groups of some extra-tileable simplices of groups satisfy assumptions of Theorem 5.6 and Theorem 5.8.

At the moment the only 7-systolic groups of virtual cohomological dimension above two known to us are the groups acting on normal 7-systolic pseudomanifolds, constructed in [13].

Question Can groups acting geometrically on normal 7-systolic pseudomanifolds of dimension at least 3 split over free nonabelian groups ?

\section{References}

[1] M Bestvina, G Mess, The boundary of negatively curved groups, J. Amer. Math. Soc. 4 (1991) 469-481 MR1096169

[2] B H Bowditch, Cut points and canonical splittings of hyperbolic groups, Acta Math. 180 (1998) 145-186 MR1638764

[3] M R Bridson, A Haefliger, Metric spaces of non-positive curvature, Grundlehren der Mathematischen Wissenschaften 319, Springer, Berlin (1999) MR1744486 
[4] M Coornaert, T Delzant, A Papadopoulos, Géométrie et théorie des groupes, Lecture Notes in Mathematics 1441, Springer, Berlin (1990) MR1075994 Les groupes hyperboliques de Gromov, with an English summary

[5] R J Daverman, Hereditarily aspherical compacta and cell-like maps, Topology Appl. 41 (1991) 247-254 MR1135101

[6] R J Daverman, A N Dranishnikov, Cell-like maps and aspherical compacta, Illinois J. Math. 40 (1996) 77-90 MR1386313

[7] J Dymara, D Osajda, Boundaries of right-angled hyperbolic buildings, Fund. Math. 197 (2007) 123-165

[8] E Ghys, P de la Harpe (editors), Sur les groupes hyperboliques d'après Mikhael Gromov, Progress in Mathematics 83, Birkhäuser, Boston (1990) MR1086648Papers from the Swiss Seminar on Hyperbolic Groups held in Bern, 1988

[9] M Gromov, Hyperbolic groups, from: "Essays in group theory”, Math. Sci. Res. Inst. Publ. 8, Springer, New York (1987) 75-263 MR919829

[10] F Haglund, Complexes simpliciaux hyperboliques de grande dimension, Prepublication Orsay 71 (2003)

[11] W Jakobsche, Homogeneous cohomology manifolds which are inverse limits, Fund. Math. 137 (1991) 81-95 MR1113561

[12] T Januszkiewicz, J Świątkowski, Nonpositively curved developments of billiards, submitted

[13] T Januszkiewicz, J Świątkowski, Simplicial nonpositive curvature, Publ. Math. Inst. Hautes Études Sci. (2006) 1-85 MR2264834

[14] T Januszkiewicz, J Świątkowski, Filling invariants of systolic complexes and groups, Geom. Topol. 11 (2007) 727-758 MR2302501

[15] I Kapovich, N Benakli, Boundaries of hyperbolic groups, from: "Combinatorial and geometric group theory (New York, 2000/Hoboken, NJ, 2001)", (S Cleary, R Gilman, A G Myasnikov, V Shpilrain, editors), Contemp. Math. 296, Amer. Math. Soc., Providence, RI (2002) 39-93 MR1921706

[16] M Kapovich, B Kleiner, Hyperbolic groups with low-dimensional boundary, Ann. Sci. École Norm. Sup. (4) 33 (2000) 647-669 MR1834498

[17] D Osajda, Connectedness at infinity of systolic complexes and groups, Groups Geom. Dyn. 1 (2007) 183-203 MR2319456

[18] P Przytycki, J Świątkowski, Flag-no-square triangulations and Gromov boundaries in dimension 3, submitted

[19] J R Stallings, On torsion-free groups with infinitely many ends, Ann. of Math. (2) 88 (1968) 312-334 MR0228573

[20] P Zawiślak, The Pontriagin sphere as a boundary of systolic groups, in preparation 
Instytut Matematyczny, Uniwersytet Wrocławski

pl. Grunwaldzki 2/4, 50-384 Wrocław, Poland

Institut de Mathématiques de Jussieu, Université Paris 6 Case 247, 4 Place Jussieu, 75252 Paris Cedex 05, France

dosaj@math.uni.wroc.pl

Received: 4 April 2007 